\title{
CANCER
}

\section{A bilateral murine tumor model to predict response to immunotherapy}

Chen, I.X. et al. PNAS 117, 23684-23694 (2020)

Over the past decade, immune checkpoint blockade (ICB) has emerged as a powerful therapeutic tool against cancer. However, many patients do not respond to ICB and the lack of robust biomarkers for predicting response to therapy remains a major issue. In a new study published in PNAS, investigators led by Rakesh Jain from the E.L. Steele Laboratories in Massachusetts General Hospital used a bilateral tumor implantation approach to explore the mechanisms underlying resistance or response to anti-PD-1 antibody in a murine orthotopic E0771 breast cancer (BC) model.

The same number of tumor cells was implanted at two distinct locations in each mouse. One tumor was removed and analysed before treatment or at early time points following it-before outcome can be assessed-to identify predictive biomarkers of response, while the second nonresected tumor was monitored to determine if the mouse responded to ICB. This model is built on the observation that the two mammary tumors implanted within the same mouse exhibit similar tumor progression or regression after treatment with anti-PD-1 antibody.

Flow cytometry and immunofluorescence analyses of the resected tumors showed that tumors that responded to ICB treatment have increased $\mathrm{CD} 8^{+} \mathrm{T}$ cell infiltration and reduced numbers of $\mathrm{CD} 11 \mathrm{~b}^{+} \mathrm{Gr} 1^{+}$ myeloid-derived suppressor cells compared to nonresponding tumors. RNA-seq on sorted $\mathrm{CD}^{+} \mathrm{T}$ cells revealed distinct gene signatures between tumors, with $\mathrm{CD}^{+}$ cells from responding tumors being highly enriched in genes from pathways related to $T$ cell activation. Altogether these findings demonstrate that responding tumors have a more immunostimulatory tumor microrenvironment compared to nonresponding tumors.

The results of the different analyses also suggested that chemokine receptor CXCR3 is important for triggering antitumor immunity in the tumor microenvironment. To validate the role of CXCR3, investigators implanted E0771 tumor cells into $\mathrm{Cxcr3^{-/ }}$ or wild-type mice before anti-PD-1 antibody treatment. Loss of CXCR3 resulted in a reduction in the number of mice responding to $\mathrm{ICB}$, thereby confirming the role of CXCR3 in antitumor immunity and highlighting its potential as a biomarker to predict response to ICB.

"These findings demonstrate the value of this 'resection and response' tumor model for identification of novel and clinically relevant biomarkers to predict response to ICB therapy and guide the use of combination therapies for BC and other cancers," write the investigators in their report.

Alexandra Le Bras

Published online: 12 October 2020

https://doi.org/10.1038/s41684-020-00669-9

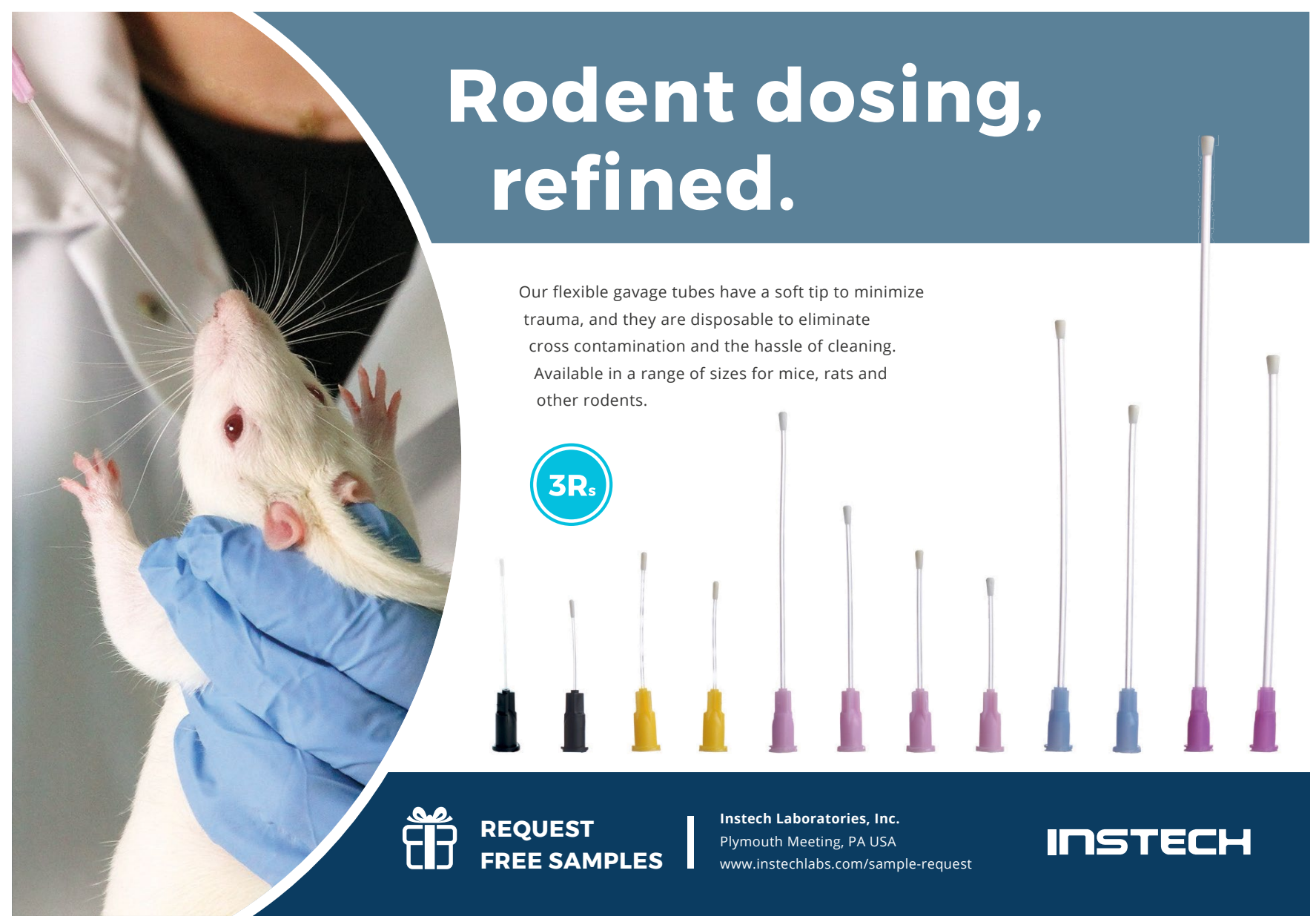

